\title{
Eine außergewöhnliche Therapie der Vena-iliaca-externa-Hypoplasie bei einer Patientin mit Klippel-Trénaunay-Syndrom - ein Fallbericht
} A unique approach in the management of external vena iliaca
hypoplasia in a patient with Klippel-Trénaunay Syndrome:
case report

Autor

Anna Ewa Cyrek

Institut

Sektion Gefäßchirurgie und Endovaskuläre Chirurgie, Klinik für Allgemein-, Viszeral- und Transplantationschirurgie, Universitätsklinikum Essen

Schlüsselwörter

Hypoplasie der Vena iliaca, Angiodysplasie,

Klippel-Trénaunay-Syndrom, Palma-Bypass

Key words

vena iliaca hypoplasia, congential angiodysplasia, Klippel-Trénaunay syndrome, Palma procedure

Bibliografie

Phlebologie 2020; 49: 299-304

Online-Publikation: 25.5 .2020

DOI 10.1055/a-1134-8807

ISSN 0939-978X

(c) 2020. Thieme. All rights reserved.

Georg Thieme Verlag KG, Rüdigerstraße 14,

70469 Stuttgart, Germany

Korrespondenzadresse

Dr. med. Anna E. Cyrek, MHBA

Sektion Gefäßchirurgie und Endovaskuläre Chirurgie

Klinik für Allgemein-, Viszeral- und Transplantationschirurgie Universitätsklinikum Essen, Hufelandstraße 55, 45147 Essen, Deutschland

Tel.: ++ 49/2 01/72311 10

Fax: ++ 49/2 01/7 23/58 05

anna.cyrek@uk-essen.de

\section{ZUSAMMENFASSUNG}

Das Klippel-Trénaunay-Syndrom (KTS) ist eine seltene und komplexe Fehlbildung. Sie zeigt die typische Trias aus vaskulärer Malformation, Knochen- und Weichteilhypertrophie sowie venöser Varikosis. Die Systemmanifestationen werden in einem individuell variablen Muster beobachtet. Eine isolierte angeborene Hypoplasie des venösen Systems tritt jedoch in den seltensten Fällen auf. Aus diesem Grund spielt die Früherkennung eine wichtige Rolle für die weitere Behandlungsplanung. So können durch rechtzeitige Behandlung schwere Krankheitsverläufe oder Spätfolgen vermieden werden. Eine isolierte angeborene Hypoplasie ist extrem selten und prädisponiert insbesondere im jungen Erwachsenenalter für Becken- oder Beinvenenthrombosen. Bei dieser Krankheit wird selten eine chirurgische Rekonstruktion durchgeführt. Wir beschreiben einen sehr seltenen Fall einer 60-jährigen Frau mit KTS, die vor 28 Jahren einen venösen CrossoverBypass (Palma) mittels Vena saphena magna bei Hypoplasie der Vena iliaca externa rechts erhalten hat. Das Ziel des vorliegenden Berichts ist das klinische Erscheinungsbild, den diagnostischen Prozess und das Krankheitsmanagement vorzustellen sowie eine Literaturübersicht zum operativen Behandlungsverfahren darzustellen.

\section{ABSTRACT}

Klippel-Trénaunay syndrome (KTS) is a rare and complex malformation. It shows the typical triad of vascular malformation, bone and soft tissue hypertrophy and venous varicosis. The system manifestations are observed in an individually variable pattern. However, isolated congenital hypoplasia of the venous system rarely occurs. For this reason, early detection plays an important role in further treatment planning. This makes it possible to avoid a severe course of disease or late sequelae by timely treatment. Isolated congenital hypoplasia is extremely rare and predisposes to pelvic or leg vein thrombosis, especially in young adults. With this disease, surgical reconstruction is rarely performed.

We describe a very rare case of a 60-year-old woman with KTS who received a venous crossover bypass (Palma procedure) 28 years ago using left graft saphenous vein with hypoplasia of the right external iliac vein. The clinical presentation, diagnostic process, and approach to management along with a literature review on the operative management are presented in this case report. 


\section{Einleitung}

Das Klippel-Trenaunay-Syndrom (KTS) ist eine seltene angeborene Erkrankung, die durch ein flaches Hämangiom der Haut (portwinestain), eine Weichteil- und Knochenhypertrophie der Extremitäten sowie eine Fehlbildung der Venen und Lymphgefäße charakterisiert ist [1]. Dabei ist die rechte untere Extremität häufiger als die linke untere Extremität betroffen. Seltener kommen oligosymptomatische, bilaterale, alternierende und gekreuzt dissoziierte Manifestationen vor [2].

Die Ätiopathogenese ist ungeklärt. Es handelt sich wahrscheinlich um eine latente Mutation. Gemäß der Hamburg-Klassifikation wird eine hämolymphatische, trunkuläre Fehlbildung mit venösen, arteriellen und lymphatischen Komponenten, die sowohl Gefäßobstruktionen als auch -dilatationen aufweisen können, diskutiert [3].

Klinisch können die Symptome nach dem Ausmaß der vorliegenden Malformationen variieren. Die Diagnose dieser embryologischen Fehlbildung ist rein klinisch. In der Diagnostik können eine duplexsonografische und angiografische Darstellung der Gefäße in betroffenen Arealen sowie radiologische Diagnostik der betroffenen Areale bei Weichteil- und Knochenhypertrophie helfen.

Für dieses komplexe Syndrom existiert keine einheitliche Therapie. Die Behandlung von KTS ist konservativ, sofern keine Komplikationen auftreten [1]. Die wichtigste konservative Maßnahme ist eine Kompressionstherapie zur Vermeidung einer chronischvenösen Insuffizienz und thromboembolischer Ereignisse [4]. Eine chirurgische Therapie im Sinne einer venösen Rekonstruktion ist umstritten und wird aufgrund der hohen Rezidivrate nicht empfohlen.

Wir beschreiben einen sehr seltenen Fall einer 60-jährigen Frau mit KTS, die vor 28 Jahren einen venösen Crossover-Bypass mittels Vena saphena magna (Palma) bei Hypoplasie der Vena iliaca externa rechts erhalten hat. Das Ziel des vorliegenden Berichts ist das klinische Erscheinungsbild, den diagnostischen Prozess und den Managementansatz sowie eine Literaturübersicht zum operativen Management vorzustellen.

\section{Fallbericht}

\section{Anamnese}

Wir berichten über eine 60-jährige Patientin, die sich im Alter von 32 Jahren mit massiver Schwellung und starken Schmerzen im Bereich des rechten Beins vorstellte. Zum damaligen Untersuchungszeitpunkt bestanden keine Ulzerationen. In der Anamnese konnten bereits im Alter von 16 Jahren wiederkehrende oberflächliche Ulzerationen am medialen distalen Unterschenkel mit Beteiligung der Innenknöchelregion ohne erkennbare Ursachen erhoben werden. Zudem kam es nach der Schwangerschaft zu einer deutlichen Zunahme der Schwellung und Schmerzen der Beine.

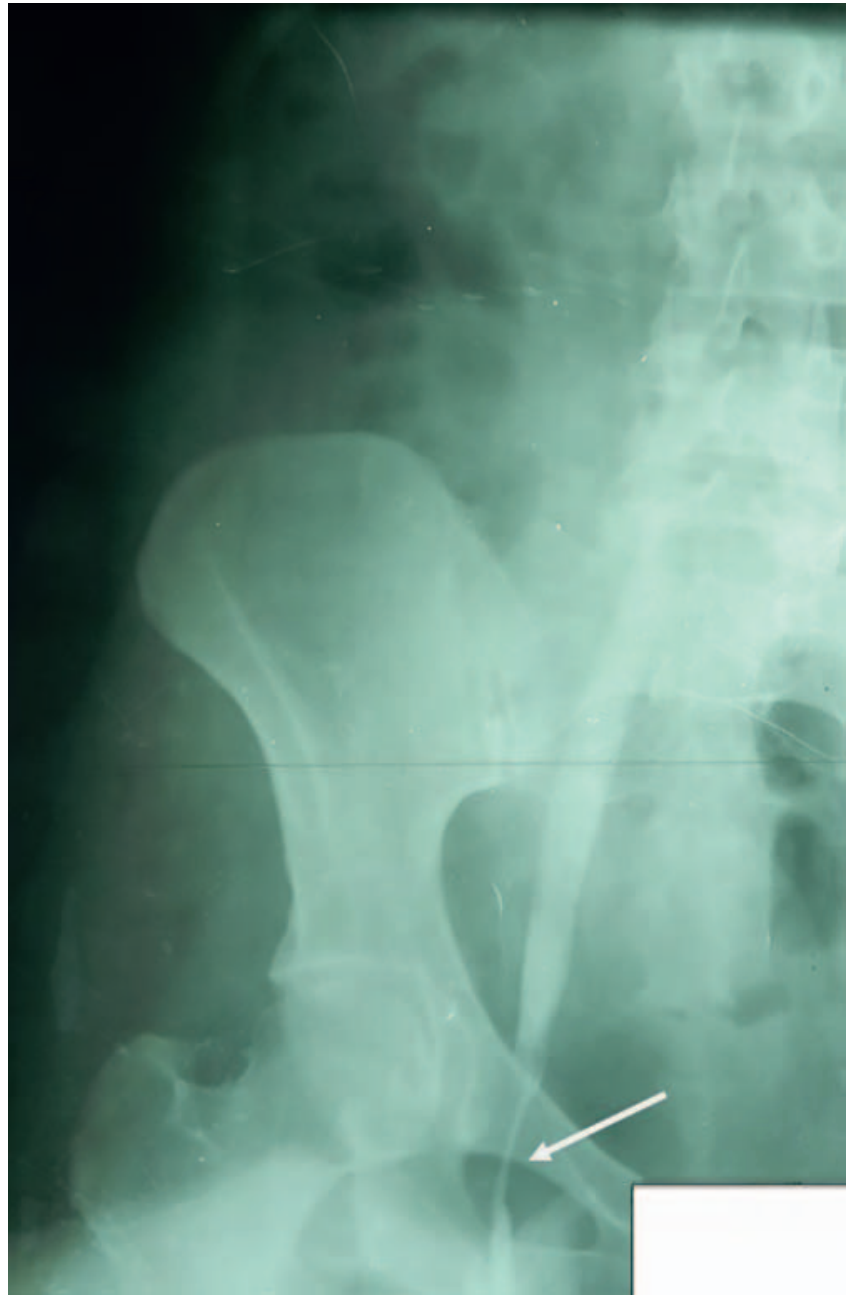

Abb. 1 Phlebografie mit Hypoplasie der rechten Vena iliaca externa ohne Zeichen einer Thrombose ( $\rightarrow$ Pfeil markiert).

\section{Befunde und Therapie}

Die körperliche Untersuchung ergab laterale Varizen und ein kleines flächiges Naevus flammeus am lateralen mittleren Oberschenkel des rechten Beins. Zusätzlich bestand eine Umfangsdifferenz zwischen der betroffenen und der kontralateralen Extremität mit vergrößertem Oberschenkel- und Wadenumfang ohne Beinlängen- oder Fußlängendifferenz. Die durchgeführten umfangreichen serologischen Untersuchungen waren unauffällig. Die angiologische Untersuchung mittels Duplexsonografie zeigte keine Insuffizienz der V. saphena parva oder V. saphena magna. Die Femoralarterien sowie peripheren Pulse waren beidseits gut tastbar. Bei einer Phlebografie zeigte sich eine Hypoplasie der rechten Vena iliaca externa ohne Zeichen einer Thrombose ( $\triangleright$ Abb. 1). Hinweise für das Vorliegen eines arteriovenösen (AV) Shunts fanden sich nicht.

Basierend auf diesem Befund sowie der typischen Trias wurde die Verdachtsdiagnose eines KTS gestellt. Um eine Thrombose sowie ein postthrombotisches Syndrom zu verhindern, wurde eine klassische Palma-Operation mit Transposition der kontralateralen Vena saphena magna als venöser Crossover-Bypass durchgeführt ( $\triangleright$ Abb. 2). Sowohl der eigentliche Eingriff als auch der postopera- 


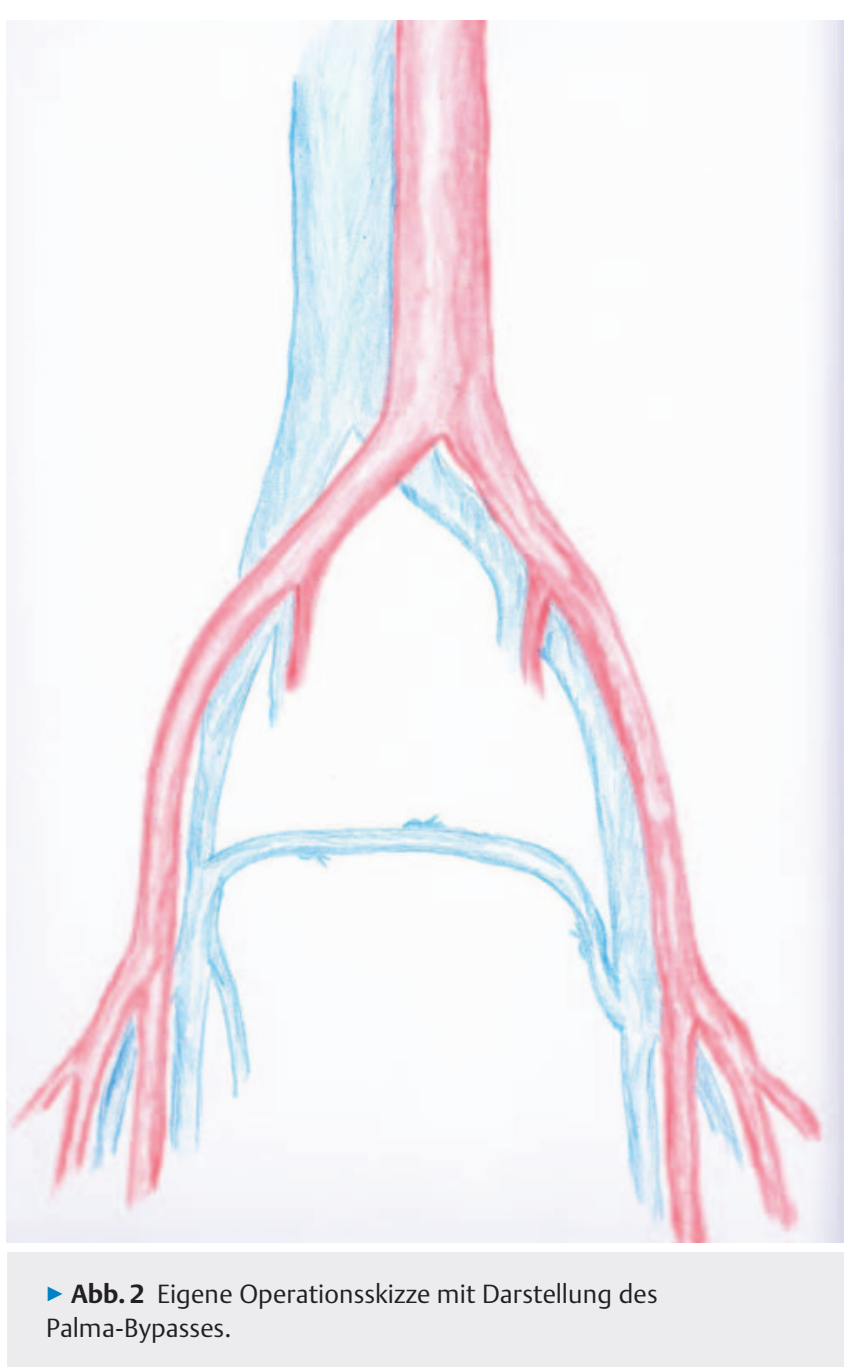

tive Verlauf gestalteten sich komplikationsfrei. Während der stationären Behandlung wurde die Patientin mit niedrigmolekularem Heparin in prophylaktischer Dosis therapiert und ohne lebenslange Antikoagulation, lediglich mit Kompressionstherapie der Klasse II nach Maß entlassen.

Bei der jetzigen Nachuntersuchung 28 Jahre nach Erstoperation hatte die Patientin keine Ödeme, Hautveränderungen oder Varikose ( $\triangleright$ Abb. $\mathbf{3}$ ) und war völlig beschwerdefrei. Es zeigten sich reizlose Narbenverhältnisse. Anamnestisch konnte bereits ein Jahr nach der Palma-Operation eine deutliche Besserung der Beschwerden festgestellt werden. In der aktuell durchgeführten venösen CT-Angiografie kam eine gute Kontinuität des Palma-Bypasses ohne Anzeichen für Stenose oder Aneurysma zur Darstellung ( $\triangleright$ Abb.4a, b).

\section{Diskussion}

Das KTS ist eine seltene Erkrankung, die häufiger unilateral (85\%) als bilateral (12,5\%) auftritt, wobei die verbleibenden 2,5\% bilateral gekreuzt auftreten können (d.h. rechte obere Extremität und linke untere Extremität) [5]. Schmerzen, Beinlängenunterschiede, Erysipel, Venenthrombose, Lungenembolie und gastrointestinale

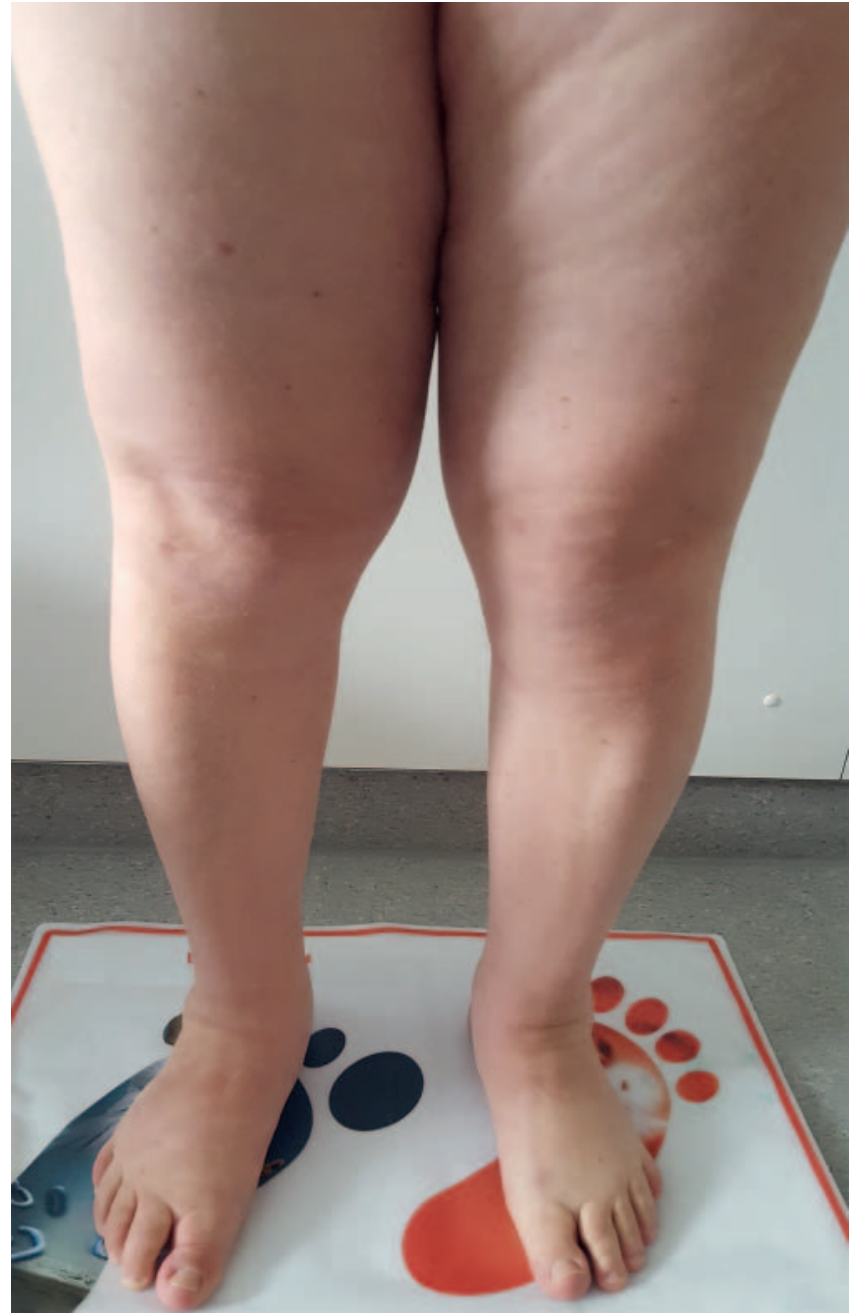

- Abb. 3 Follow-up 28 Jahre nach der Palma-Operation: keine Ödeme, Hautveränderungen oder Varikose.

Blutungen sind typische klinische Symptome [6]. Die Gefäßveränderungen betreffen meist die Kapillaren im Sinne eines Naevus flammeus sowie das Venensystem als Krampfadern, aber auch in Form einer Hypo- oder Aplasie der tiefen Extremitätenvenen $[5,6]$. Die Diagnose kann gestellt werden, wenn 2 der folgenden Befunde vorliegen: Kapillarmissbildungen (port-winestain), eine Beinlängendifferenz aufgrund von Weichteil- und Knochenhypertrophie sowie eine atypische, meist laterale oberflächliche Varikose [7]. Differenzialdiagnostisch sollten bei diesen Patienten immer auch andere Angiodysplasien ausgeschlossen werden. Für die Differenzierung kann die sogenannte Hamburger Klassifikation der International Society for the Study of Vascular Anomalies (ISSVA) herangezogen werden [8].

Die venösen Missbildungen werden bei 68-80\% der Patienten beobachtet $[9,10]$. Die Ätiologie dieser Entität ist jedoch unbekannt. Es werden verschiedene Theorien diskutiert. Einerseits wird vermutet, dass die Krankheit aufgrund einer Dysfunktion in den sympathischen Ganglien entsteht. Andererseits wird die These vertreten, dass die Anomalien im tiefen venösen System über Thrombosen entstehen, die über eine venöse Hypertonie zu einer Extremitätenhypertrophie führen [1,6]. Des Weiteren wird über 

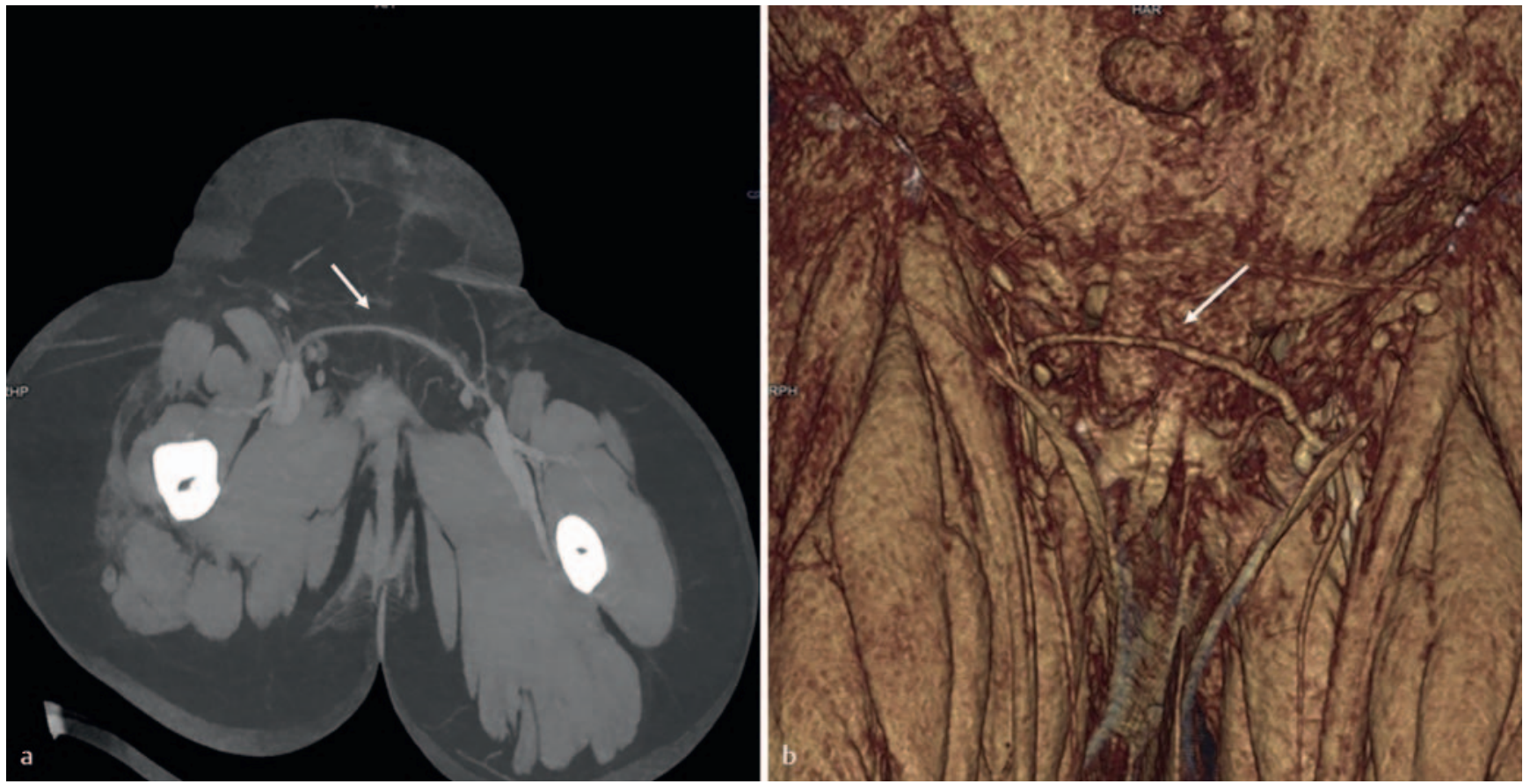

- Abb.4 Venöse CT-Angiografie 28 Jahre nach der Implantation eines femoro-femoralen Crossover-Bypasses (Palma) bei einer 60-jährigen Patientin mit Klippel-Trénaunay-Syndrom ( $\rightarrow$ Pfeil markiert: Darstellung des Palma-Bypasses). a Projektions-CT-Bild mit maximaler Intensität. b 3D-CT-Bild mit guter Kontinuität des Palma-Bypasses ohne Anzeichen für Stenose oder Aneurysma.

die Relevanz für die VG5Q-Mutation des kurzen Chromosomenarms im Gefäßgen diskutiert [5]. Ein weiterer möglicher Mechanismus wäre eine primäre Obstruktion des Venensystems, welche zur venösen Hypertonie und zu Veränderungen der Angiogenese sowie einer Vaskulogenese auf dem Boden diverser genetischer Prädispositionen, führen kann [11]. Fetale Tierstudien haben gezeigt, dass sich diese embryonalen Venen im Laufe von 12 Lebenswochen bilden und zurückbilden. Ergebnisse zu Studien am Menschen liegen jedoch nicht vor [12].

Die Diagnose des KTS ist klinisch schwierig zu stellen, da es keinen echten pathognomonischen Test gibt. Anamneseerhebung, ausführliche klinische Untersuchung und die Ultraschalluntersuchung sind wichtige Maßnahmen für die erste Diagnosesicherung. Doppler- und farbkodierte Duplexsonografie sind hilfreich für die Beurteilung der Durchblutungssituation ("fast-flow" versus "slowflow"). Duplexultraschall, Magnetresonanztomografie (MRT) und Computertomografie (CT) können eine Weichteilhypertrophie und damit verbundene Gefäßmissbildungen darstellen. Die Phlebografie wird als hilfreich für die präoperative Planung angesehen, wenn Verfahren zur Eliminierung oberflächlicher Venen, wie Sklerotherapie, Ligatur oder Stripping, geplant sind. Wenn zuvor noch keine Dokumentation eines tiefen Venensystems durchgeführt wurde, kann auch hier die Phlebografie hilfreich sein [13].

Die Behandlung von KTS-Patienten besteht hauptsächlich aus einer konservativen medizinischen Behandlung, einschließlich Kompressionstherapie und entzündungshemmenden Medikamenten zur Schmerzlinderung. Ziel der Therapie dieser angeborenen Erkrankung ist es, Beschwerden zu mindern, mögliche Beeinträchtigungen von funktionellem Gewebe und daraus resultierende Komplikationen zu verhindern und Patienten somit zu mehr
Lebensqualität zu verhelfen. Da es bei einer inkompletten, nicht indizierten oder falschen Therapie nicht selten zu einer Verschlechterung der Symptome kommt, ist eine strenge Risiko-NutzenAbwägung vor jeder interventionellen oder operativen Therapie erforderlich.

Die hämodynamische Einteilung der Gefäßmalformationen bestimmt das jeweilige Therapieverfahren [14]. Die Mehrzahl der Gefäßmalformationen kann minimalinvasiv durch interventionelle Verfahren wie Sklerosierungsbehandlungen (Slow-flow-Malformationen) und Katheter-gestützte Embolisationen (Fast-flowMalformationen) therapiert werden [9, 10, 14, 15]. Offene Rekonstruktionen sollten nur dann erwogen werden, wenn eine oberflächliche Lokalisation einen sicheren Zugangsweg ermöglicht. Aufgrund der Affektion mehrerer Gewebeschichten und dem erhöhten Blutungsrisiko gestaltet sich die Präparation technisch schwierig (die Gefäßwände sind dysplastisch und fragil) und sollte somit Spezialisten vorbehalten bleiben.

Eine isolierte angeborene Hypoplasie ist extrem selten und prädisponiert insbesondere im jungen Erwachsenenalter für Becken- oder Beinvenenthrombosen. Die chirurgische Therapie im Sinne einer venösen Rekonstruktion wird kontrovers diskutiert und ist Patienten mit intakten tiefen Venensystemen vorbehalten, die nicht für eine weniger invasive Behandlung infrage kommen (Lasertherapie, Sklerotherapie, Schaumsklerotherapie mit Natriumtetra-Decylsulfat und Polidocanol) [15]. In unserem Fall erfolgte die Implantation eines veno-venösen Palma-Bypasses. Bei dieser Technik wird die kontralaterale Vena saphena magna für einen Crossover-Bypass verwendet, um die venöse Stauung in den betroffenen Extremitäten zu dekomprimieren [16]. Die Vene wird subkutan im suprapubischen Raum zur betroffenen Seite 
getunnelt und von einem Ende zum anderen mit der V. femoralis communis anastomosiert. Die Anastomose kann je nach Anatomie und Ausmaß der venösen Obstruktion auf die Vena saphena magna oder die Vena femoralis communis ausgedehnt werden. Ein wichtiger Faktor für die Durchgängigkeit des Transplantats ist der anhaltende Fluss bei ausreichendem Druck. Einige Autoren schlugen die vorteilhaften Wirkungen einer temporären arteriovenösen Fistel vor, um die Durchblutung der Venentransplantate zu erhöhen [17, 18]. Andere zeigten, dass die Durchgängigkeit ohne die Verwendung einer arteriovenösen Fistel erreicht werden kann [19]. In unserem Fall erhielt die Patientin keine arteriovenöse Fistelbildung. Selbst ohne eine arteriovenöse Fistel zu erzeugen, war die Durchgängigkeit während der Nachbeobachtungszeit ausgezeichnet.

In der heutigen Zeit besteht durch die Entwicklung endovaskulärer Therapieverfahren grundsätzlich die Möglichkeit zur minimalinvasiven endovenösen Therapie des KTS. Belege für die Effektivität dieser Therapieansätze stehen jedoch aus. Die in das Venensystem eingesetzten Stents und Transplantate weisen üblicherweise eine höhere Thromboserate auf als arterielle Stents oder Transplantate. Eine kürzlich durchgeführte Überprüfung legt nahe, dass ein verringerter Fluss in die V. femoralis communis der beste Prädiktor für eine In-Stent-Stenose oder einen Transplantatverschluss ist [20].

Des Weiteren sind die Patienten mit KTS mit einer geringgradigen Koagulopathie assoziiert [21] und haben ein höheres Risiko für thromboembolische Erkrankungen [22]. In der klinischen Bewertung wurde jedoch keine Effektivität einer Antikoagulation nachgewiesen. Eine Indikation hierfür besteht bei Patienten, die eine tiefe Venenthrombose entwickelten oder eine venöse Intervention erhielten [1]. Physiotherapeutische Maßnahmen, insbesondere die Kompressionstherapie mit medizinischen Kompressionsstrümpfen oder Kompressionsverbänden, sind zwingend erforderlich.

Über die Prognose der Erkrankung sind keine detaillierten Angaben in der Literatur vorhanden. Sie hängt im Wesentlichen vom Diagnosezeitpunkt, der individuellen Ausprägung der Systemmanifestationen und einem möglichst frühen Therapiebeginn ab [1].

\section{Fazit}

Die Diagnose und Behandlung des KTS kann aufgrund der geringen Inzidenz schwierig sein. Trotz der Variabilität der Erkrankung ist die einheitliche Diagnose eine absolute Voraussetzung für eine erfolgreiche Therapie. Dabei ist die Ausprägung der Gefäßdysplasien an der jeweiligen Lokalisation entscheidend für das individuelle therapeutische Vorgehen.

Unser Fallbericht lässt die klinische Bedeutung des Klippel-Trénaunay-Syndroms aus praktischer Sicht erkennen und weist auf die Erkennung der korrekten Diagnose und die damit verbundenen Konsequenzen für die richtige Therapie hin.

\section{Ethische Richtlinien}

Die für dieses Manuskript erhobenen Daten stimmen mit den nationalen Gesetzen und der aktuellen Helsinki-Deklaration überein, eine Einverständniserklärung der Patientin lag vor.

\section{HINWEIS}

Diese Kasuistik wurde bei folgenden Veranstaltungen als Poster präsentiert:

33. Jahrestagung der Deutschen Gesellschaft für Gefäßchirurgie, 27.09.-30.09.2017, Frankfurt am Main, Deutschland Klinischer Erfolg 24 Jahre nach einem veno-venösen Bypass (Palma) bei Hypoplasie der Vena iliaca

Fourth Annual Congress of the European Society for Vascular Medicine, 18.03.-20.03.2018, Prague, Czech Republic

A unique approach in the management of vena iliaca hypoplasia in a patient with Klippel-Trenaunay syndrome

Interessenkonflikt

Die Autorinnen/Autoren geben an, dass kein Interessenkonflikt besteht.

Literatur

[1] Jacob AG, Driscoll D], Shaughnessy W] et al. Klippel-Trenaunay syndrome: spectrum and management. Mayo Clinic Proceedings 1998; 73 (1): $28-36$

[2] Lee A, Driscoll D, Gloviczki P et al. Evaluation and management of pain in patients with Klippel-Trenaunay syndrome: a review. Pediatrics 2005; 115 (3): 744-749

[3] Belov S, Loose DA, Weber J. Vascular Malformations, Periodica Angiologica 16. Reinbek: Einhorn Presse Verlag; 1989: 25-30

[4] Gloviczki P, Driscoll D]. Klippel-Trenaunay syndrome: current management. Phlebology 2007; 22: 291-298

[5] Redondo P, Aguado L, Martinez-Cuesta A. Diagnosis and management of extensive vascular malformations of the lower limb. Part I. Clinical diagnosis. J Am Acad Dermatol 2011; 65: 893-906

[6] Uihlein LC, Liang MG, Fishman S] et al. Capillary-venous malformation in the lower limb. Pediatr Dermatol 2013; 30: 541-548

[7] Mavili E, Ozturk M, Akcali Y et al. Direct CT venography for evaluation of the lower extremity venous anomalies of Klippel-Trenaunay syndrome. Am J Roentgenol 2009; 192: 311-316

[8] Wassef M, Blei F, Adams D et al. Vascular Anomalies Classification: Recommendations from the International Society for the Study of Vascular Anomalies. Pediatrics 2015; 136: e203-e214

[9] Kim YW, Lee BB, Cho JH et al. Haemodynamic and clinical assessment of lateral marginal vein excision in patients with a predominantly venous malformation of the lower extremity. Eur J Vasc Endovasc Surg 2007; 33 : 122-127

[10] Cherry K], Gloviczki P, Stanson AW. Persistent sciatic vein: diagnosis and treatment of a rare condition. J Vasc Surg 1996; 23: 490-497

[11] Zea MI, Hanif M, Habib M et al. Klippel-Trenaunay syndrome: a case report with brief review of literature. J Dermatol Case Rep 2009; 3: 56-59

[12] Oduber CE, Young-Afat DA, van der Wal AC et al. The persistent embryonic vein in Klippel-Trénaunay syndrome. Vasc Med 2013; 18: 185191

[13] Mavili E, Ozturk M, Akcali Y et al. Direct CT venography for evaluation of the lower extremity venous anomalies of Klippel-Trenaunay syndrome. Am J Roentgenol 2009; 192: 311-316

[14] Wohlgemuth WA, Müller-Wille R, Teusch VI et al. The retrograde transvenous pushthrough method: a novel treatment of peripheral arterio- 
venous malformations with dominant venous outflow. Cardiovasc Intervent Radiol 2015; 38: 623-631

[15] Cabrere J, Cabrera J Jr, Olmeda AG et al. Treatment of Venous Malformations With Sclerosant in Microfoam Form. Arch Dermatol 2003; 139 : 1409-1416

[16] Power AH, Gloviczki P. Left iliac vein occlusion treated with a Palma procedure. J Vasc Surg Venous Lymphat Disord 2014; 2: 204-205

[17] Alimi YS, Dinauro P, Fabre D et al. Iliac vein reconstructions to treat acute and chronic venous occlusive disease. J Vasc Surg 1997; 25 (4): 673-681

[18] Plate G, Hollier LH, Gloviczki P et al. Overcoming failure of venous vascular prostheses. Surgery 1984; 96 (3): 503-510

[19] Halliday P, Harris J, May J. Femoro-femoral crossover grafts (Palma Operation): A long-term follow-up study. In: Bergan J], Yao JS, editors Surgery of the Veins. Orlando: Grune \& Stratton; 1985: 241-254
[20] Kurstjens R, de Wolf M, Kleijnen J et al. The predictive value of haemodynamic parameters for outcome of deep venous reconstructions in patients with chronic deep vein obstruction - A systematic review. Phlebology 2017; 32 (8): 532-542

[21] Mason KP, Neufeld EJ, Karian VE et al. Coagulation abnormalities in pediatric and adult patients after sclerotherapy or embolization of vascular anomalies. American Journal of Roentgenology 2001; 177 (6): 13591363

[22] Huiras EE, Barnes C], Eichenfield LF et al. Pulmonary thromboembolism associated with Klippel-Trenaunay syndrome. Pediatrics 2005; 116 (4): e596-e600 